\title{
A CLASS OF PERIODIC ORBITS OF SUPERIOR PLANETS*
}

\author{
BY \\ F. R. MOULTON

\section{Introduction.}

The periodic solutions of the problem of three bodies fall into many classes requiring somewhat different modes of treatment. One of these classes $\dagger$ is that in which a small, or infinitesimal, body moves near another of finite mass and is subject to the disturbing influence of a distant large mass which moves in a circle about the finite mass except in so far as it is disturbed by the small body. Moreover, the orbit of the small body is circular except as it is disturbed by the distant large mass.

In this paper the problem of a distant infinitesimal body moving subject to the attraction of two finite masses relatively near each other and revolving about their common center of gravity in circles is considered, and the existence of certain periodic solutions is established. As the finite bodies approach coincidence the orbit of the distant infinitesimal body approaches a circle. The restriction that the distant mass shall be infinitesimal is no more necessary than it was in the earlier problem, but it is made for the sake of simplicity.

The mode of treatment here is similar to that adopted in the earlier investigation. For the value zero of the parameter $\mu$ the problem reduces to that of two bodies which admits a circular orbit as a periodic solution. The existence of the analytic continuation of this orbit as a periodic solution is proved, and direct methods of constructing the series are developed. It is shown how the integral can be used as a check on the computations, or to replace one of the differential equations in the construction of the solutions.

The earlier paper had direct and important applications to the Lunar Theory and to the satellite theory in general. The results of this paper do not find applications in the solar system, except possibly in the case of the most remote small satellites of Jupiter and Saturn which are subject to the attractions of the central planets and the larger interior satellites, but they have some use in certain triple star systems. Their chief value is certainly that they cover a part

\footnotetext{
* Read before the American Mathematical Society, Chicago, April 29, 1911. This paper was written while the author was a Research Associate of the Carnegie Institution of Washington.

† Treated by the writer in unese Transactions, vol. 7 (1906), pp. 537-577. 
of the field of periodic orbits of the restricted problem of three bodies, and an examination of the whole field is necessary for the discussion of the evolution of these orbits with a continuous change of the parameters upon which they depend, for example the constant of the Jacobian integral which Dr. HrLL and Sir George Darwin adopted in their important researches.* The results of such an analysis as is given here supplement the laborious numerical investigations of HILL and DARwIN.

\section{The differential equations.}

Let $m_{1}$ and $m_{2}$ represent the masses of the finite bodies and $k^{2}$ the gravitational constant. Suppose the infinitesimal body is projected in the plane of motion of the finite bodies; its orbit will be a plane curve. Let the origin of coördinates be the center of gravity of the system and the $x y$-plane the plane of motion. Let the coördinates of $m_{1}, m_{2}$, and the infinitesimal body be $x_{1}, y_{1} ; x_{2}, y_{2}$; and $x, y$ respectively. Then the differential equations of motions for the infinitesimal body are

$$
\begin{gathered}
\frac{d^{2} x}{d t^{2}}=\frac{\partial U}{\partial x}, \quad \frac{d^{2} y}{d t^{2}}=\frac{\partial U}{\partial y}, \\
U=\frac{k^{2} m_{1}}{r_{1}}+\frac{k^{2} m_{2}}{r_{2}}, \\
r_{1}=\sqrt{\left(x-x_{1}\right)^{2}+\left(y-y_{1}\right)^{2}}, \quad r_{2}=\sqrt{\left(x-x_{2}\right)^{2}+\left(y-y_{2}\right)^{2}} .
\end{gathered}
$$

Let

$$
r=\sqrt{x^{2}+y^{2}}, \quad R=\sqrt{\left(x_{1}-x_{2}\right)^{2}+\left(y_{1}-y_{2}\right)^{2}}
$$

$$
R_{1}=\sqrt{x_{1}^{2}+y_{1}^{2}}=\frac{m_{2}}{m_{1}+m_{2}} R, \quad R_{2}=\sqrt{x_{2}^{2} \pm y_{2}^{2}}=\frac{m_{1}}{m_{1}+m_{2}} R
$$

Then equations (1) become in polar coördinates

$$
\begin{aligned}
& \frac{d^{2} r}{d t^{2}}-r\left(\frac{d v}{d t}\right)^{2}=\frac{\partial U}{\partial r} \\
& r \frac{d^{2} v}{d t^{2}}+2 \frac{d r}{d t} \frac{d v}{d t}=\frac{1}{r} \frac{\partial U}{\partial v}
\end{aligned}
$$

We now develop the potential function $U$. From (1) and (2) we find

- Hill, American Journal of Mathematics, vol. 1 (1878), pp. 5-26, 129-147, 245260; Darwin, Acta Mathematica, vol. 21 (1897), pp. 99-242; Mathematische Annalen, vol. 51 (1898-9), pp. 523-583. 


$$
\begin{aligned}
U= & \frac{k^{2} m_{1}}{r}\left[1-\frac{2 R_{1}}{r} \cos \left(v-v_{1}\right)+\left(\frac{R_{1}}{r}\right)^{2}\right]^{-\frac{1}{2}} \\
& \quad+\frac{k^{2} m_{2}}{r}\left[1+\frac{2 R_{2}}{r} \cos \left(v-v_{1}\right)+\left(\frac{R_{2}}{r}\right)^{2}\right]^{-1} \\
= & \frac{k^{2}\left(m_{1}+m_{2}\right)}{r}+\frac{k^{2} m_{1} m_{2}}{m_{1}+m_{2}} \frac{R^{2}}{r^{3}}\left\{1\left[1+3 \cos 2\left(v-v_{1}\right)\right]\right. \\
+\frac{R_{1}-R_{2}}{8 r} & {\left.\left[3 \cos \left(v-v_{1}\right)+5 \cos 3\left(v-v_{1}\right)\right]+\cdots\right\} . }
\end{aligned}
$$

Then equations (3) become

$$
\begin{aligned}
\frac{d^{2} r}{d t^{2}}-r\left(\frac{d v}{d t}\right)^{2}+\frac{k^{2}\left(m_{1}+m_{2}\right)}{r^{2}}=-\frac{k^{2} m_{1} m_{2}}{m_{1}+m_{2}} \frac{R^{2}}{r^{4}}\left\{\frac{3}{4}\left[1+3 \cos 2\left(v-v_{1}\right)\right]\right. \\
\left.+\frac{R_{1}-R_{2}}{2 r}\left[3 \cos \left(v-v_{1}\right)+5 \cos 3\left(v-v_{1}\right)\right]+\cdots\right\},
\end{aligned}
$$

(5)

$$
\begin{aligned}
r \frac{d^{2} v}{d t^{2}}+2 \frac{d r}{d t} \frac{d v}{d t}= & -\frac{k^{2} m_{1} m_{2}}{m_{1}+m_{2}} \frac{R^{2}}{r^{4}}\left\{\frac{3}{2} \sin 2\left(v-v_{1}\right)\right. \\
& \left.+\frac{3\left(R_{1}-R_{2}\right)}{8 r}\left[\sin \left(v-v_{1}\right)+5 \sin 3\left(v-v_{1}\right)\right]+\cdots\right\} .
\end{aligned}
$$

If the orbits of $m_{1}$ and $m_{2}$ are circles, as is assumed to be the case, equations (1) admit the Jacobian integral *

where

$$
\left(\frac{d x}{d t}\right)^{2}+\left(\frac{d y}{d t}\right)^{2}-2 n_{1}\left[x \frac{d y}{d t}-y \frac{d x}{d t}\right]=2 U-C,
$$

$$
n_{1}=\frac{k \sqrt{m_{1}+m_{2}}}{R^{i}}, \quad C=\text { constant of integration. }
$$

It follows that $n_{1}$ is the mean motion of the finite bodies and that $v_{1}=n_{1}\left(t-t_{0}\right)$. The integral becomes in polar coördinates

$$
\left(\frac{d r}{d t}\right)^{2}+r^{2}\left(\frac{d v}{d t}\right)^{2}-2 n_{1} r^{2} \frac{d v}{d t}=2 U-C .
$$

When the right members are neglected equations (5) have the particular solution

$$
r=a, \quad v=\frac{k \sqrt{m_{1}+m_{2}}}{a^{3}}\left(t-t_{0}\right)=n\left(t-t_{0}\right),
$$

where $n$ is the angular velocity of the infinitesimal body in its orbit and $t_{0}$ is an arbitrary constant. It will be supposed that $n$ is given by the observations,

* See Moulton's Introduction to Celestial Mechanics, p. 202. 
or that its value is assumed, and that $a$ is determined by the second equation of (8). The constant $a$ has three determinations, only one of which is real.

New variables $\rho, \theta$, and $\tau$ and new constants $\mu$ and $M$ will be introduced by the equations

$$
\begin{gathered}
r=a(1+\rho), \quad v=n\left(t-t_{0}\right)+\theta, \\
\frac{n}{n_{1}}=\mu, \quad\left(n-n_{1}\right)\left(t-t_{0}\right)=\tau, \\
\frac{m_{1} m_{2}}{\left[m_{1}+m_{2}\right]^{2}}=M .
\end{gathered}
$$

It follows from (6), (8), and (9) that

$$
\frac{R}{a}=\mu^{3} .
$$

Then equations (5) become

$$
\begin{aligned}
\frac{d^{2} \rho}{d \tau^{2}}-(1+\rho)\left[\frac{\mu}{1-\mu}-\frac{d \theta}{d t}\right]^{2}+\frac{\mu^{2}}{(1-\mu)^{2}} \frac{1}{(1+\rho)^{2}} \\
=\frac{-M \mu^{32}}{(1-\mu)^{2}} \frac{(1+\rho)^{4}}{\left(1+\frac{3}{4}\right.}[1+3 \cos 2(\tau+\theta] \\
\left.\quad+\frac{m_{2}-m_{1}}{m_{1}+m_{2}} \frac{\mu^{3}}{2(1+\rho)}[3 \cos (\tau+\theta)+5 \cos 3(\tau+\theta)]+\cdots\right\}, \\
(1+\rho) \frac{d^{2} \theta}{d \tau^{2}}+2 \frac{d \rho}{d \tau}\left[\frac{\mu}{1-\mu}-\frac{d \theta}{d \tau}\right]=\frac{-M \mu^{42}}{(1-\mu)^{2}(1+\rho)^{4}}\left\{\frac{3}{2} \sin 2(\tau+\theta)\right. \\
\left.\quad+\frac{m_{2}-m_{1}}{m_{1}+m_{2}} \frac{3 \mu^{3}}{8(1+\rho)}[\sin (\tau+\theta)+5 \sin 3(\tau+\theta)]+\cdots\right\} .
\end{aligned}
$$

These equations are valid for the determination of the motion of the infinitesimal so long as $|\mu|<1$. The right member of the first one involves only cosines, and of the second one only sines, of integral multiples of $(\tau+\theta)$. The parts in the brackets proceed according to powers of $\mu^{3}$, the coefficients of even powers of $\mu^{3}$ in the first and second equations being cosines and sines respectively of even multiples of $(\tau+\theta)$, and the coefficients of odd powers of $\mu^{3}$ being cosines and sines of odd multiples of $(\tau+\theta)$.

\section{Existence of periodic solutions.}

Suppose $\rho=\beta, d \rho / d t=0, \theta=0, d \theta / d \tau=\gamma$ at $\tau=0$ and let the solution of (11) be written in the form

$$
\begin{aligned}
& \rho=f(\beta, \gamma ; \tau), \\
& \theta=\varphi(\beta, \gamma ; \tau) .
\end{aligned}
$$


Now make the transformation

$$
\rho=\rho^{\prime}, \quad \theta=-\theta^{\prime}, \quad \tau=-\tau^{\prime} .
$$

The resulting equations have precisely the form (11). Consequently their solutions with the initial conditions $\rho^{\prime}=\beta, d \rho^{\prime} / d \tau=0, \theta^{\prime}=0, d \theta^{\prime} / d \tau=\gamma$ are

$$
\begin{aligned}
& \rho^{\prime}=f\left(\beta, \gamma ; \tau^{\prime}\right)=f(\beta, \gamma ;-\tau)=\rho, \\
& \theta^{\prime}=\varphi\left(\beta, \gamma ; \tau^{\prime}\right)=\varphi(\beta, \gamma ;-\tau)=-\theta .
\end{aligned}
$$

Therefore, w.th the initial conditions adopted, $\rho$ is an even function of $\tau$ and $\theta$ is an odd function of $\tau$. The orbit is symmetrical both geometrically and in $\tau$ with respect to an axis passing through the origin and rotating with any constant velocity. In particular, it is symmetrical with respect to the line joining the finite bodies. Such an orbit will be called symmetrical whether it is periodic or not.

Now consider the conditions for a closed symmetrical orbit. Since the right members of (11) involve sines and cosines of integral multiples of $\tau$, sufficient conditions that $\rho$ and $\theta$ in symmetrical orbits shall be periodic with the period $2 j \pi, j$ an integer, are

$$
\begin{aligned}
d \rho / d \tau & =f^{\prime}(\beta, \gamma ; j \pi)=0, \\
\theta & =\varphi(\beta, \gamma ; j \pi)=0 .
\end{aligned}
$$

These conditions are necessary provided they are distinct.

In order to examine the solutions of (15) it is convenient to use other parameters than $\beta$ and $\gamma$. Suppose that at $\tau=0$

$$
\begin{gathered}
r=a(1+\rho)=a(1+\alpha)(1-e), \\
\frac{d r}{d \tau}=a \frac{d \rho}{d t}=0, \quad \theta=0, \\
\frac{\mu}{1-\mu}-\frac{d \theta}{d t}=\frac{\mu}{1-\mu} \frac{V \overline{1+e}}{(1+\alpha) \cdot(1-e)} .
\end{gathered}
$$

It follows that $a(1+\alpha)$ and $e$ are the major semi-axis and the eccentricity of the elliptic orbit which we should obtain if the right members of equations (11) were zero. Because of the well-known properties of the solutions in terms of these elements, the properties of the general solutions so far as they do not depend upon the right members of (11) are known. These properties will be important in solving the conditions for periodic solutions.

Equations (11) are regular in the vicinity of $\mu=0, \rho=0, d \rho / d t=0$, $\theta=0, d \theta / d \tau=0$ for all $\tau$. It follows from Cauchy's general existence theorem on the solutions of differential equations that the solutions are analytic functions 
of $\alpha, e$, and $\mu^{3}$ which are regular in the vicinity of $\alpha=e=\mu^{3}=0$; and it follows from Poincare's extension * of Cauehy's results that the moduli of $\alpha$, $e$, and $\mu^{\xi}$ can be taken so small that the solutions are regular while $\tau$ runs through any finite preassigned range of values. We shall choose as the interval for $\tau$ the range $0 \leqq \tau \leqq 2 j \pi$ and integrate (11) as power series in $\alpha, e$, and $\mu^{3}$, vanishing with $\alpha=e=\mu^{\xi}=0$. That is, our results are the analytic continuation with respect to these parameters of the particular solution $r=a, v=n t$ which we have when $\mu=0$. The results may be written in the form

$$
\begin{gathered}
\rho=p_{1}\left(\alpha, e, \mu^{\sharp} ; \tau\right), \\
\frac{d \rho}{d t}=p_{2}\left(\alpha, e, \mu^{\natural} ; \tau\right), \\
\theta=p_{3}\left(\alpha, e, \mu^{\natural} ; \tau\right), \\
\frac{d \theta}{d \tau}=p_{4}\left(\alpha, e, \mu^{\natural} ; \tau\right),
\end{gathered}
$$

where $p_{1}, \cdots, p_{4}$ are power series in $\alpha, e, \mu^{3}$ with $\tau$ entering in the coefficients.

The conditions (15) for a symmetric periodic solution become

$$
\begin{aligned}
& p_{2}\left(\alpha, e, \mu^{\mathbf{3}} ; j \pi\right)=0, \\
& p_{3}\left(\alpha, e, \mu^{\mathbf{j}} ; j \pi\right)=0 .
\end{aligned}
$$

It will be shown that these equations can be solved for $\alpha$ and $e$ as power series in $\mu^{3}$, vanishing with $\mu^{3}=0$, and converging for the modulus of $\mu^{j}$ sufficiently small.

Since the right members of (11) carry $\mu^{4}$ as a factor, the part of the solution depending on the right members will be divisible by $\mu^{3 p}$. If the right members of (11) were zero and the solution were formed with the initial conditions.(16), the mean angular motion of the infinitesimal body in its orbit would be

$$
\nu=\frac{\mu}{(1-\mu)(1+\alpha)^{1}} .
$$

Consequently it follows from the solution of the two-body problem that equations (18) have the form

$$
\begin{aligned}
& p_{2}(j \pi)=e \nu\{\sin \nu j \pi+e \sin 2 v j \pi+\cdots\}+\mu^{4 \vartheta} q_{2}\left(\alpha, e, \mu^{3} ; j \pi\right)=0, \\
& p_{3}(j \pi)=\left\{\frac{\mu}{1-\mu} j \pi-\nu j \pi-2 e \sin \nu j \pi-\cdots\right\}+\mu^{48} q_{3}\left(\alpha, e, \mu^{3} ; j \pi\right)=0,
\end{aligned}
$$

where the unwritten parts in the \{\} are sines of multiples of $\nu j \pi$ and carry $e^{2}$ as a factor.

\footnotetext{
* Acta Mathematica, vol. 13 (1890), p. 16.
} 
On referring to (19) it is seen that the first equation of (20) is divisible by $\mu^{2}$ and the second by $\mu$. After division by these factors the equations are still satisfied by $\alpha=e=\mu=0$. Moreover the determinant of their linear terms in $\alpha$ and $e$ is

$$
\Delta=\mid \begin{array}{cc}
0, & j \pi \\
{ }_{2}^{3} j \pi, & -2 j \pi
\end{array}=-\frac{3}{2} j^{2} \pi^{2} \neq 0 .
$$

Therefore, besides the solution $\mu=0$, equations (20) have a unique solution for $\alpha$ and $e$ as power series in $\mu^{\frac{1}{3}}$, vanishing with $\mu^{\frac{1}{3}}=0$, which converge for the modulus of $\mu^{\frac{1}{3}}$ sufficiently small. These power series carry $\mu^{4}$ as a factor and can be written

$$
\alpha=\mu^{\frac{4}{3}} P_{1}\left(\mu^{\frac{1}{3}}\right), \quad e=\mu^{\frac{4}{3}} P_{2}\left(\mu^{\frac{1}{3}}\right) .
$$

On substituting these series in the right members of (17), which vanish with $\alpha=e=\mu^{\frac{3}{3}}=0$, we have

$$
\rho=\mu^{\frac{3}{3}} Q_{1}\left(\mu^{3} ; \tau\right), \quad \theta=\mu^{\frac{4}{3}} Q_{2}\left(\mu^{\frac{1}{3}} ; \tau\right) .
$$

The series $Q_{1}$ and $Q_{2}$ are periodic in $\tau$ with the period $2 j \pi$ because the conditions that the solutions shall have this period have been satisfied. Since (17) converge for all $0 \leqq \tau \leqq 2 j \pi$ if the moduli of $\alpha, e$, and $\mu^{\frac{1}{3}}$ are sufficiently small, and since the expressions for $\alpha$ and $e$ given in (22) vanish for $\mu=0$, it follows that the modulus of $\mu^{\frac{3}{3}}$ can be taken so small that the series (23) converge for all $\tau$ in the interval; and since they are periodic with the period $2 j \pi$, the convergence holds for all finite values of $\tau$.

The integer $j$ has so far been undetermined. When $j$ is unity the periodic solutions exist uniquely and their period is $2 \pi$. When $j$ is greater than unity the periodic solutions also exist uniquely. Since the periodic orbits for $j$ greater than unity include those for $j$ equal to unity, and since in both cases there is precisely one periodic orbit for a given value of $\mu^{\frac{1}{3}}$, it follow's that all the symmetrical periodic orbits of the class under consideration have the period $2 \pi$.

It follows from (6) and (9) that $\tau+\theta=v-v_{1}$. Since in the periodic solution $\theta$ is periodic with the period $2 \pi$, the period of the solution is the synodic period of the three bodies. Hence, if we refer the motion of the infinitesimal body to a set of axes having their origin at the center of gravity of the system and rotating in the direction of motion of the finite bodies at the angular rate at which they move, and if the $x$-axis passes through the finite bodies, the periodic orbit of the infinitesimal body, which has been proved to exist, will be symmetrical with respect to the $x$-axis. Since by hypothesis $a>R$ it follows from (6) and (8) that $n_{1}>n$. Therefore even if the motion of the infinitesimal body is forward with respect to fixed axes it is retrograde with respect to the rotating axes. 
It is supposed that the period of the finite bodies, and therefore $n_{1}$, is given in advance and remains fixed. The variation of the parameter $\mu^{\frac{3}{3}}$ corresponds to a variation of the period of the infinitesimal body defined by $n$. If the motion with respect to fixed axes is forward $n$ has the same sign as $n_{1}$ and $\mu^{\frac{1}{3}}$ has three values, one of which is real and positive while the other two are complex. If the motion is retrograde $\mu^{3}$ has three different values, one of which is real and negative while the other two are complex. Therefore for a given period there are six symmetrical orbits, three direct and three retrograde, and for small $|\mu|$ one direct orbit is real and one retrograde orbit is real, while in the others the coördinates are complex. This means, of course, that the corresponding solutions do not exist in the physical problem. The coördinates of the complex orbits are conjugate in pairs. For a certain value of $\mu^{\frac{1}{3}}$ they may become equal, and therefore real, and for larger values of $\mu^{\frac{3}{3}}$ real and distinct, as Darwin found by numerical computations to be true for satellites in a special case in A c t a M a the m a t i c a, vol. 21 (1897), pp. 99-242.

Transforming the integral (7) by (9), we find

$$
\begin{aligned}
& \left(\frac{d}{d \tau} \frac{\rho}{d \tau}\right)^{2}+(1+\rho)^{2}\left[\frac{\mu}{1-\mu}-\frac{d \theta}{d \tau}\right]^{2}-\frac{(1+\rho)^{2}}{1-\mu}\left[\frac{\mu}{1-\mu}-\frac{d \theta}{d \tau}\right]=\frac{\mu^{2}}{(1-\mu)^{2}(1+\rho)} \\
& +\frac{2 M \mu^{\frac{20}{3}}}{(1-\mu)^{2}(1+\rho)^{3}}\left\{\frac{1}{4}[1+3 \cos 2(\tau+\theta)]\right. \\
& \left.+\frac{m_{2}-m_{1}}{m_{1}+m_{2}} \frac{\mu^{3}}{8(1+\rho)}[3 \cos (\tau+\theta)+5 \cos 3(\tau+\theta)]+\cdots\right\}-C_{1},
\end{aligned}
$$

where $C=n_{1}^{2}(1-\mu)^{2} a^{2} C_{1}$. It follows from this equation and (23) that $C_{1}$ can be expanded as a power series in $\mu^{3}$, vanishing with $\mu^{3}=0$. The term of lowest degree in $\mu^{\frac{3}{3}}$ after substituting (23) is $\mu^{\frac{3}{3}}$. Therefore $\mu^{\frac{3}{3}}$ can be expanded as a power series in $C_{1}^{\ddagger}$. For $C_{1}=0$ the three branches of the function are the same. Since $a=R / \mu^{\frac{3}{3}}$ the relation between $C$ and $C_{1}$ is

$$
C=\frac{n_{1}^{2} R^{2}(1-\mu)^{2} C_{1}}{\mu^{3}}=\frac{n_{1}^{2} R^{2}}{\mu^{3}}[1+\text { power series in } \mu] \text {. }
$$

From this it follows that $C=\infty$ for $\mu^{\frac{3}{3}}=0$. Therefore the periodic orbits branch at $C=\infty$, and there are two cycles of three each.

\section{Practical construction of the periodic solutions.}

It has been proved that the symmetrical periodic solutions under discussion are expressible in the form

$$
\rho=\sum_{i=4}^{\infty} \rho_{i} \mu^{i / 3}, \quad . \quad \theta=\sum_{i=4}^{\infty} \theta_{i} \mu^{i / 3},
$$


where the $\rho_{i}$ and $\theta_{i}$ are functions of $\tau$. Since these series are periodic and converge for all $\left|\mu^{3}\right|$ sufficiently small, it follows that each $\rho_{i}$ and $\theta_{i}$ is separately periodic; that is,

$$
\rho_{i}(\tau+2 \pi) \equiv \rho_{i}(\tau), \quad \theta_{i}(\tau+2 \pi) \equiv \theta_{i}(\tau) .
$$

In every closed orbit there are points at which $d \rho / d \tau=0$. We shall suppose that $t_{0}$ of $(9)$ is so determined that this condition is satisfied at $\tau=0$. It follows. from this and the convergence of (9) for all $\left|\mu^{\frac{1}{3}}\right|$ sufficiently small that

$$
\frac{d \rho_{i}}{d t}=0 \quad(j=4, \cdots, \infty) .
$$

In the symmetrical periodic orbits whose existence has been proved above we have also $\theta=0$ at $\tau=0$. But we shall not now impose this condition because the general periodic orbits include those which are symmetrical; and if in the construction it appears that the conditions for symmetry are a consequence of those for periodicity, we shall have proved that all the periodic orbits of the class under consideration are symmetrical. Such will be seen to be the case.

Equations (26) are to be substituted in (11), arranged as power series in $\mu^{\mathbf{k}}$, and the coefficients of the several powers of $\mu^{\boldsymbol{k}}$ set equal to zero. The coefficients. of $\mu^{3}$ set equal to zero give the equations

$$
\frac{d^{2} \rho_{4}}{d \tau^{2}}=0, \quad \frac{d^{2} \theta_{4}}{d \tau^{2}}=0 .
$$

The solutions of these equations satisfying (27) and (28) are

$$
\rho_{4}=a_{4}, \quad \theta_{4}=b_{4},
$$

where $a_{4}$ and $b_{4}$ are constants which are so far undetermined.

The coefficients of $\mu^{l}, \cdots, \mu^{3}$ are the same as (29) except for their subscripts, and their solutions satisfying (27) and (28) are similarly

$$
\rho_{j}=a_{i}, \quad \theta_{i}=b_{j}
$$$$
(j=5, \cdots, 9),
$$

where the $a_{j}$ and $b_{j}$ are all undetermined constants.

The coefficients of $\mu^{*}$ give the equations

$$
\begin{aligned}
& \frac{d^{2} \rho_{10}}{d \tau^{2}}=3 a_{4}-\frac{3 M}{4}[1+3 \cos 2 \tau], \\
& \frac{d^{2} \rho_{10}}{d \tau^{2}}=-\frac{3 M}{2} \sin 2 \tau .
\end{aligned}
$$

In order that the solution of the first of these equations shall be periodic we must impose the condition

$$
a_{4}=\frac{M}{4}
$$


which uniquely determines the constant $a_{4}$. Then the solutions of (32) satisfying (27) and (28) are

$$
\begin{aligned}
& \rho_{10}=a_{10}+\frac{9}{16} M \cos 2 \tau, \\
& \theta_{10}=b_{10}+\frac{8}{8} M \sin 2 \tau,
\end{aligned}
$$

$a_{10}$ and $b_{10}$ remaining undetermined.

The coefficients of $\mu^{4}$ are

$$
\frac{d^{2} \rho_{11}}{d \tau^{2}}=3 a_{5}, \quad \frac{d^{2} \theta_{11}}{d \tau^{2}}=0,
$$

from which it follows that

$$
a_{5}=0, \quad \rho_{11}=a_{11}, \quad \theta_{11}=b_{11},
$$

where $a_{11}$ and $b_{11}$ are as yet undetermined.

The coefficients of $\mu^{3 / 2}$ are

$$
\begin{aligned}
& \frac{d^{2} \rho_{12}}{d \tau^{2}}=3 a_{6}+\frac{M\left(m_{1}-m_{2}\right)}{2\left(m_{1}+m_{2}\right)}[3 \cos \tau+5 \cos 3 \tau], \\
& \frac{d^{2} \theta_{12}}{d \tau^{2}}=\frac{3}{8} \frac{M\left(m_{1}-m_{2}\right)}{m_{1}+m_{2}}[\sin \tau+5 \sin 3 \tau] .
\end{aligned}
$$

Imposing conditions (27) and integrating, we find

$$
\begin{aligned}
a_{6} & =0, \\
\rho_{12} & =a_{12}-\frac{M\left(m_{1}-m_{2}\right)}{2\left(m_{1}+m_{2}\right)}\left[3 \cos \tau+\frac{5}{8} \cos 3 \tau\right], \\
\theta_{12} & =b_{12}-\frac{3 M\left(m_{1}-m_{2}\right)}{8\left(m_{1}+m_{2}\right)}\left[\sin \tau+\frac{5}{8} \sin 3 \tau\right],
\end{aligned}
$$

where $a_{12}$ and $b_{12}$ remain undetermined.

In a similar way we find from the coefficients of $\mu^{\text {st }}$

$$
a_{7}=0, \quad \rho_{13}=a_{13}+\frac{8}{2} M \cos 2 \tau, \quad \theta_{13}=b_{13}+\frac{8}{16} M \sin 2 \tau,
$$

$a_{13}$ and $b_{13}$ remaining undetermined.

So far all the $b_{j}$ have remained arbitrary and it is necessary to carry the integration one step further in order to see how they are determined. The coefficients of $\mu^{4}$ are

$$
\begin{aligned}
& \frac{d^{2} \rho_{14}}{d \tau^{2}}=3 a_{8}-3 a_{4}^{2}+\frac{8}{2} M\left[3 b_{4} \sin 2 \tau+2 a_{4}+6 a_{4} \cos 2 \tau\right], \\
& \frac{d^{2} \theta_{14}}{d \tau^{2}}=-a_{4} \frac{d^{2} \theta_{10}}{d \tau^{2}}-3 M\left[b_{4} \cos 2 \tau-2 a_{4} \sin 2 \tau\right] .
\end{aligned}
$$


Substituting the values of $a_{4}$ and $\theta_{10}$ from (33) and (34), imposing the conditions (27) and integrating, we find

$$
\begin{aligned}
& a_{8}=-\frac{3 M^{2}}{16}, \\
& \rho_{14}=a_{14}-\frac{9}{8} b_{4} M \sin 2 \tau-\frac{9}{16} M^{2} \cos 2 \tau, \\
& \theta_{14}=b_{14}+\frac{3}{4} b_{4} M \cos 2 \tau-\frac{1}{3} \frac{5}{2} M^{2} \sin 2 \tau .
\end{aligned}
$$

Imposing the condition (28) for $j=14$, we have

$$
\begin{aligned}
b_{4} & =0, \\
\rho_{14} & =a_{14}-\frac{9}{16} M^{2} \cos 2 \tau, \\
\theta_{14} & =b_{14}-\frac{1}{3} \frac{5}{2} M^{2} \sin 2 \tau .
\end{aligned}
$$

From the coefficients of $\mu^{\frac{18}{3}}$ it is found in a similar way that

$$
\begin{aligned}
& b_{5}=0, \\
& \rho_{15}=a_{15}-\frac{8}{4} M \frac{\left(m_{1}-m_{2}\right)}{m_{1}+m_{2}}\left[\cos \tau+\frac{5}{27} \cos 3 \tau\right], \\
& \theta_{15}=b_{15}+3 M \frac{\left(m_{1}-m_{2}\right)}{m_{1}+m_{2}}\left[\sin \tau+\frac{5}{2} \sin 3 \tau\right],
\end{aligned}
$$

$a_{15}$ and $b_{15}$ remaining arbitrary.

It will be observed that so far as the computation has been carried the coefficients of the $\rho_{j}$ are cosines of integral multiples of $\tau$ and the coefficients of the $\theta_{i}$, except for the undetermined additive constants, are sines of integral multiples of $\tau$. In the computation of $\rho_{j}$ the periodicity conditions have uniquely determined $a_{j-6}$, and the condition $d \rho_{i} / d \tau=0$ at $\tau=0$ has required that $b_{j-10}=0$. It will now be shown that these properties are general. Suppose $\rho_{4}, \cdots, \rho_{n}$, $\theta_{4}, \cdots, \theta_{n}$ have been computed and that the coefficients are all known except $a_{n-5}, \cdots, a_{n}$ which enter additively in $\rho_{n-5}, \cdots, \rho_{n}$ respectively, and $b_{n-9}$, $\cdots, b_{n}$ which enter additively in $\theta_{n-9}, \cdots, \theta_{n}$ respectively. For the determination of $\rho_{n+1}$ and $\theta_{n+1}$ we have

$$
\begin{aligned}
& \frac{d^{2} \rho_{n+1}}{d \tau^{2}}=3 a_{n-5}+\frac{9}{2} M b_{n-9} \sin 2 \tau+F_{n+1}(\tau), \\
& \frac{d^{2} \theta_{n+1}}{d \tau^{2}}=G_{n+1}(\tau),
\end{aligned}
$$

where $F_{n+1}(\tau)$ and $G_{n+1}(\tau)$ are entirely known functions of $\tau$. It follows from the assumptions respecting $\rho_{4}, \cdots, \rho_{n}, \theta_{4}, \cdots, \theta_{n}$ and the properties of equation (11) that $F_{n+1}(\tau)$ is a sum of cosines of integral multiples of $\tau$ and that 
$G_{n+1}(\tau)$ is a sum of sines of integral multiples of $\tau$. Hence we may write

$$
\begin{aligned}
& F_{n+1}(\tau)=\Sigma A_{j}^{(n+1)} \cos j \tau, \\
& G_{n+1}(\tau)=\Sigma B_{j}^{(n+1)} \sin j \tau .
\end{aligned}
$$

In order that the solution of the first equation of (42) shall be periodic we must impose the condition

$$
3 a_{n-5}+A_{0}^{(n+1)}=0,
$$

which uniquely determines $a_{n-5}$.

After equation (43) has been satisfied the solution of the first equation of $(42)$ is

$$
\begin{aligned}
\rho_{n+1} & =a_{n+1}-\frac{9}{8} M b_{n-9} \sin 2 \tau+\Sigma \alpha_{j}^{(n+1)} \cos j \tau, \\
\alpha_{j}^{(n+1)} & =-\frac{1}{j^{2}} A_{j}^{(n+1)} .
\end{aligned}
$$

The condition $d \rho / d \tau=0$ at $\tau=0$ makes it necessary to impose the condition

$$
b_{n-9}=0 .
$$

Then $\rho_{n+1}$ is completely determined except for the additive constant $a_{n+1}$, and is a surn of cosines of integral multiples of $\tau$.

The solution of the second equation of (42) is

$$
\begin{aligned}
\theta_{n+1} & =b_{n+1}+\Sigma \beta_{j}^{(n+1)} \sin j \tau, \\
\beta_{j}^{(n+1)} & =-\frac{1}{j^{2}} B_{j}^{(n+1)} .
\end{aligned}
$$

Hence $\theta_{n+1}$ is a sum of sines of integral multiples of $\tau$, except for the undetermined constant $b_{n+1}$ which must be put equal to zero in order to satisfy the conditions on $\rho_{n+11}$. These results lead, by induction, to the conclusion that the $\rho_{j}$ and $\theta_{j}, j=4, \cdots, \infty$, are sums of cosines and sines respectively of integral multiples of $\tau$ whose coefficients are uniquely determined.

It follows from the properties of the solutions which have just been established that not only is $d \rho / d \tau=0$ at $\tau=0$ but also $\theta(0)=0$. Therefore these periodic orbits are the symmetrial orbits whose existence was established in $\S 3$. In the construction it was not assumed that the orbits were symmetrical, and since this property was a necessary consequence of the periodicity conditions it follows that all periodic solutions which are expansible as power series in $\mu^{\frac{3}{3}}$ are symmetrical. It is easily shown by direct consideration of the construction of periodic solutions that they cannot be expanded as power series in $\mu^{1 / 3}$ except when $j$ is a multiple of 3 , and that then they reduce to those found above. 


\section{Application of the integral.}

The differential equations admit the integral (24) which, for brevity, can be written in the form

$$
F\left(\rho, \frac{d \rho}{d \tau}, \theta, \frac{d \theta}{d t}, \tau, \mu^{3}\right)=0
$$

It follows from the form of (24) and the expansion (26) that the left member of this equation can be developed as a power series in $\mu^{\xi}$, giving

$$
F=F_{0}+F_{1} \mu^{3}+F_{2} \mu^{3}+\cdots+F_{n} \cdot \mu^{3}+\cdots=0 .
$$

Since the $\rho_{j}$ and $\theta_{j}$ are sums of cosines and sines respectively of integral multiples of $\tau$, and since $d \rho / d \tau$ enters in (24) only in the second degree and $\theta$ only in even degrees, it follows that the $F_{j}$ are sums of cosines of integral multiples of $\tau$. Equation (47) is an identity in $\mu^{\frac{3}{3}}$, whence

$$
F_{n}=\Sigma C_{j}^{(n)} \cos j \tau=0 \quad(n=0, \ldots, \infty) .
$$

Since these equations hold for all values of $\tau$, we have

$$
C_{j}^{(n)}=0 \quad(n, j=0, \ldots, \infty) .
$$

The $C_{j}^{(n)}$ are functions of the $\alpha_{j}^{(0)}, \cdots, \alpha_{j}^{(n)}$ and $\beta j^{(0)}, \cdots, \beta_{j}^{(n)}$. Hence equations (48) can be used as check formulas on the computation of the coefficients of the solutions.

Equations (48) can be used in place of the second equation of (11) for the determination of the $\beta_{j}^{(n)}$, the coefficients of the trigonometric terms in the expression for $\theta_{n}$. Suppose $\rho_{4}, \cdots, \rho_{n-1}$ and $\theta_{4}, \cdots, \theta_{n-1}$ have been determined except for additive constants in $\rho_{n-6}, \cdots, \rho_{n-1}$. It follows from (24) that $F_{n}$ is

$$
F_{n}=\frac{d \theta_{n}}{d \tau}+P_{n}\left(\rho j, \frac{d \rho_{j}}{d \tau}, \theta_{j}, \frac{d \theta_{j}}{d \tau}\right) \quad(j=4, \ldots, n-1),
$$

where $P_{n}$ is a polynomial in the arguments indicated. Consequently equations (48) are of the form

$$
C_{j}^{(n)}=\beta_{j}^{(n)}+D_{j}^{(n)}\left(\alpha_{k}^{(\nu)}, \beta_{k}^{(\nu)}\right)=0 \quad(\nu=4, \cdots, n-1),
$$

where the $D_{j}^{(n)}$ are known polynomials in the $\alpha_{k}^{(\nu)}$ and the $\beta_{k}^{(v)}$. Therefore equations (50) uniquely determine the $\beta_{j}^{(n)}$.

The University of Chicago, April 29, 1911. 